\title{
The MURAVES muon telescope: technology and expected performances
}

\author{
Giulio Saracino ${ }^{1,2,}$, Fabio Ambrosino ${ }^{1,2}$, Lorenzo Bonechi ${ }^{3}$, Alan Bross ${ }^{4}$, Luigi Cimmino ${ }^{1}$, \\ Roberto Ciaranfi ${ }^{3}$, Raffaello D’Alessandro ${ }^{3,5}$, Flora Giudicepietro ${ }^{6}$, Giovanni Macedonio ${ }^{6}$, \\ Marcello Martini ${ }^{6}$, Vincenzo Masone ${ }^{1}$, Nicola Mori ${ }^{3,5}$, Pasquale Noli ${ }^{1}$, Massimo Orazi ${ }^{6}$, \\ Giuseppe Passeggio ${ }^{1}$, Anna Pla-Dalmau ${ }^{4}$, Lorenzo Roscilli ${ }^{1}$, Paolo Strolin ${ }^{1,2}$
}

\author{
${ }^{1}$ Istituto Nazionale di Fisica Nucleare (INFN), Sezione di Napoli, Naples, Italy \\ ${ }^{2}$ Università di Napoli Federico II, Dipartimento di Fisica, Naples, Italy \\ ${ }^{3}$ Istituto Nazionale di Fisica Nucleare (INFN), Sezione di Firenze, Sesto Fiorentino, Florence, Italy \\ ${ }^{4}$ Fermilab, Batavia, Illinois, USA \\ ${ }^{5}$ Università di Firenze, Dipartimento di Fisica e Astronomia, Sesto Fiorentino, Florence, Italy \\ ${ }^{6}$ Istituto Nazionale di Geofisica e Vulcanologia (INGV), Sezione di Napoli - Osservatorio Vesuviano, Naples, Italy
}

\author{
Article history \\ Received July 5, 2016; accepted October 5, 2016. \\ Subject classification: \\ Muon radiography, Muography, Volcanoes, Geophysical imaging, Particle detectors.
}

\begin{abstract}
The MURAVES project aims to study the inner structure of the upper part of the Mt. Vesuvius volcano by muon radiography (muography) technique. Very high energy muons, produced by cosmic rays in the atmosphere, can penetrate large thickness of rocks. By measuring the attenuation of the muons flux trough the volcano cone is possible to obtain a $2 \mathrm{D}$ image of the density structure. Internal discontinuities, with a spatial resolution of about $10 \mathrm{~m}$, can be, in principle, resolved. An absolute average density measurement can be provided too. The project, funded by the Italian Ministry of University, Research and Education (MIUR), is led by INGV and INFN. In this article the mechanical structure of the detectors and background suppression techniques are reported.
\end{abstract}

\section{Introduction}

The MURAVES project represents the first attempt for a muography of the upper part of Mt. Vesuvius. This challenging project implies a strong background rejection and a higher sensitivity with respect to previous muographies. A muon telescope consisting of an array of four identical detectors will be used, for a total sensitive area of $4 \mathrm{~m}^{2}$. The detectors are designed on the basis of the experience acquired in the MU-RAY R\&D program [Ambrosi et al. 2011; Anastasio et al. 2013a, 2013b; Ambrosino et al. 2014, 2015] using the same technology of plastic scintillator bars of triangular cross-section, equipped by wave-length shifting (WLS) fibres that are coupled to silicon photomul- tipliers (SiPMs). Major technical upgrades have been introduced in the mechanical structure, in the photo-sensors, in the front-end electronics and in DAQ Board. The last three technical upgrades are discussed in a dedicated contribution to these proceedings [Cimmino et al. 2017]. Here we will focus on the mechanical structure and present some considerations on the crucial issue of background suppression.

\section{Mechanical structure}

The basic "module" of the telescope consists of 32 plastic scintillator bars with their 32 WLS fibres conveyed to an optical connector. The optical connector is located on a printed circuit board (PCB) that hosts the 32 SiPMs. A front-end electronic board (called SLAVE) provides the voltage bias to the SiPMs and gives a local trigger (LT) digital signal when at least one of the 32 SiPMs produces a signal above the discriminator threshold. The LT signals of all modules are processed by a data acquisition board (called MASTER), which gives a global trigger (GT) signal according to its programmable trigger logic. When a GT is sent back to the SLAVE boards, all the relevant data are acquired and stored by the MASTER.

Two modules are pre-assembled side-by-side in a "layer", having about $1 \mathrm{~m}^{2}$ active area. Each layer is enclosed, equipped by the SiPMs and the SLAVE boards, 


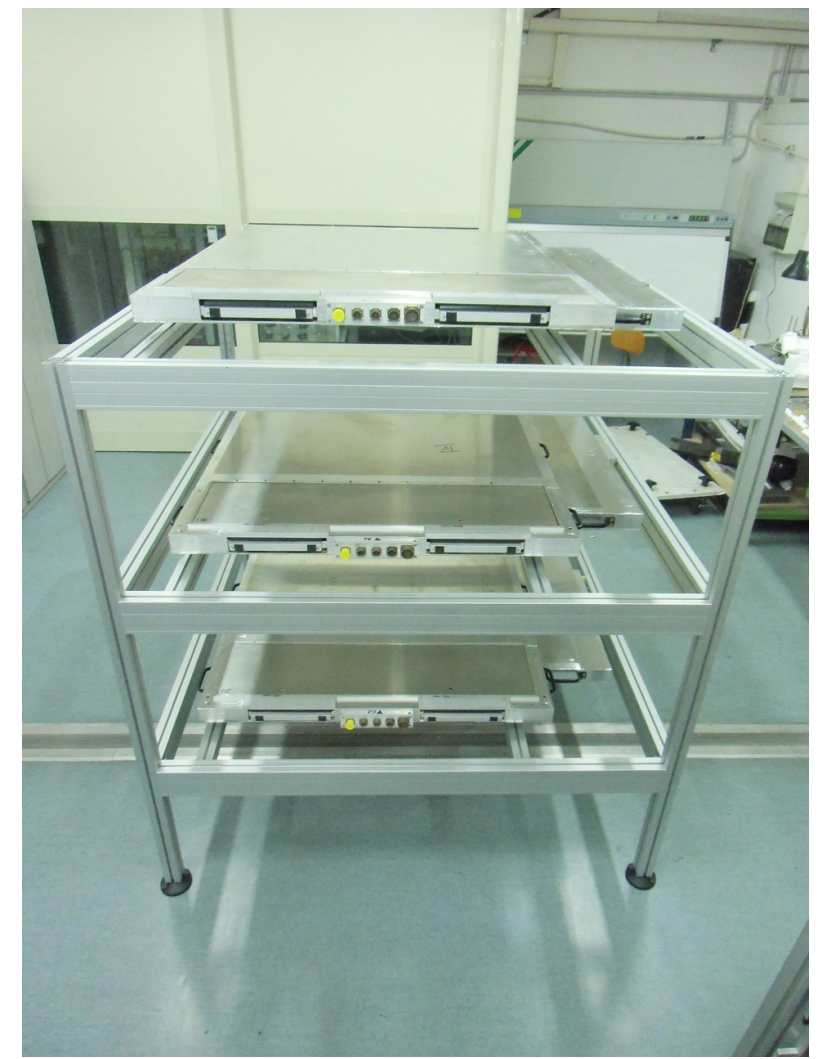

Figure 1. Three X-Y planes placed horizontally in the cosmic test stand.

in an aluminium shell that protects the modules and their electronics. The layers weigh about $60 \mathrm{~kg}$ and can be easily handled by two persons. Circular military type connectors make cabling very easy.

The layers give the coordinate perpendicular to the bars and lying in its geometrical plane. Two adjacent layers with orthogonal bars form a "X-Y plane". These planes can be placed vertically thank to special foots that can be fixed on a frame or directly on the floor. Alternatively the planes can be mounted horizontally on a support frame, as shown in Figure 1.

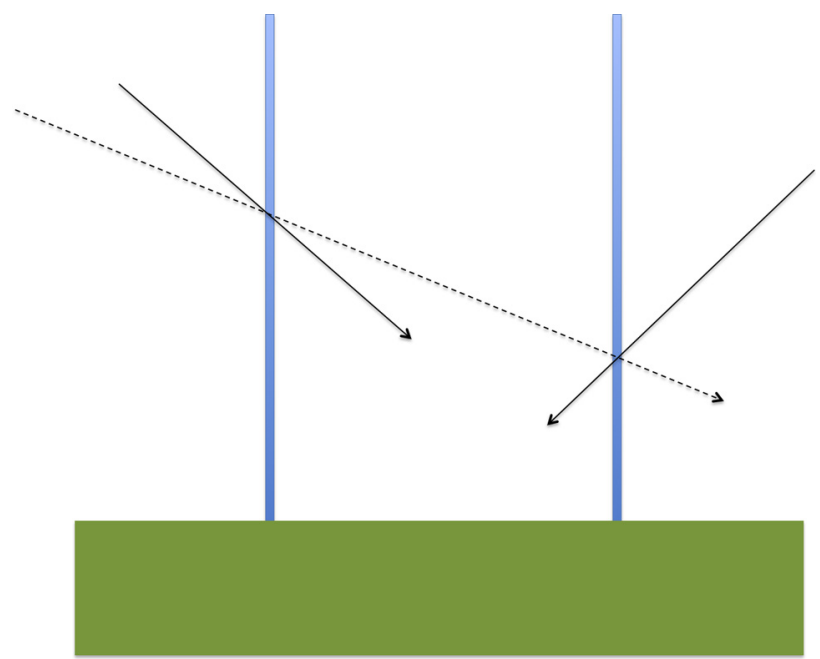

Figure 2. Example of a fake track generated by two not temporally correlated particles.
The modularity of the system allows building up muon telescopes with flexible geometry. The X-Y planes are self-sustaining and can be placed easily to different distances from each other. Since the MASTER can control up to 32 SLAVES, up to $8 \mathrm{X}$-Y planes can be connected to form a tracker with more layers and/or with a larger surface.

\section{Background sources}

The recorded events may correspond to muons that crossed the volcano coming from the observed direction, or may be due to background. A strong background rejection becomes essential for low muon fluxes, as in the case of rock thickness of order of $1 \mathrm{~km}$. The most likely sources of background are fake tracks or real tracks produced by electrons, positrons and background muons.

\section{Fake tracks}

Fake tracks are reconstructed from hits of spurious origin, either temporally correlated or not.

Temporally uncorrelated hits may originate from dark counts or from one or more uncorrelated particles, hitting different layers of the detector (Figure 2). The corresponding background can be reduced by lowering the dark count rates, by reducing the time coincidence window among the hits, or by increasing the number of X-Y planes or the spatial resolution, in order to have tighter constraints in the alignment of the track. A way to measure this background is to put out of time the signal coming from the different layers.

A fake track may result also from several particles temporally correlated, as those produced in the same cosmic shower (Figure 3). In addition to tighter constraints, a time resolution enabling to measure the time of flight of the candidate muon helps its rejection.

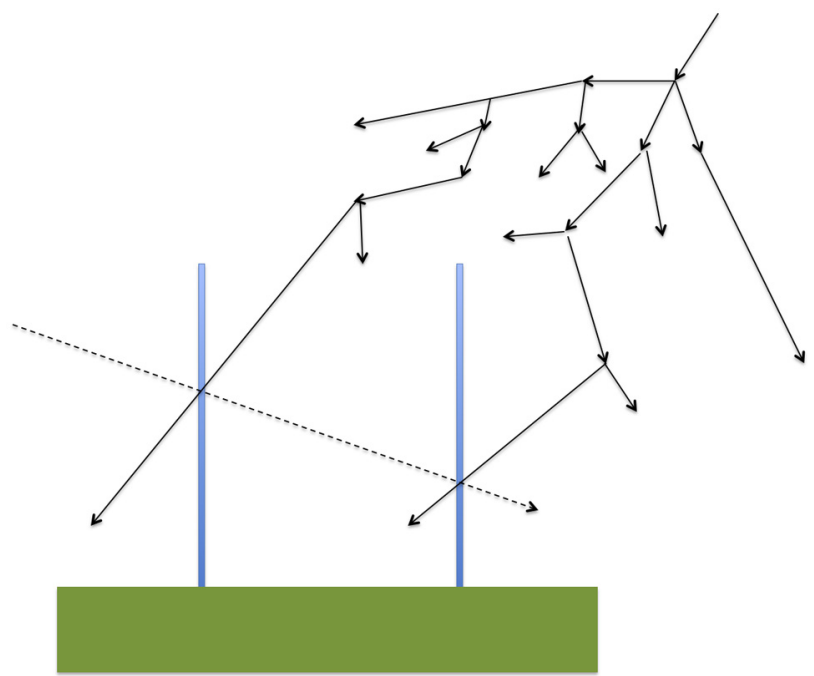

Figure 3. Example of a fake track generated by two temporally correlated particles coming from the same cosmic shower. 


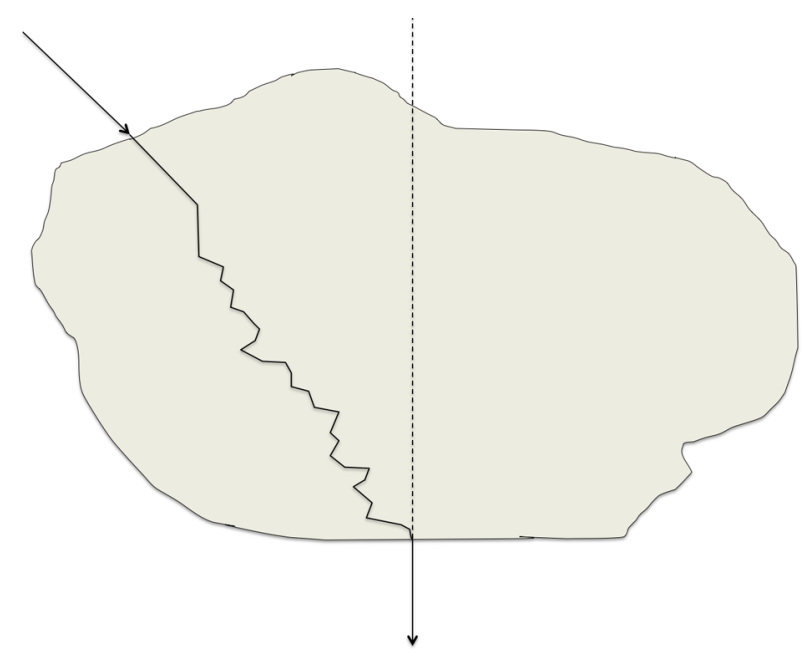

Figure 4. Pictorial view of a muon entering the volcano with a certain direction and exiting with a different one because of the multiple scattering inside the rock.

\section{Real tracks produced by electrons and positrons}

At the ground level, part of the cosmic radiation consists of electrons, positrons and gammas. Some of them may produce a track if they cross the detector in the backward-forward (BF) or forward-backward (FB) direction. A technique to reduce this background is to introduce a slab of material (typically iron) between tracking planes, so that these particles are absorbed or produce an electromagnetic shower that can be identified by the presence of high multiplicity hits. Due to the poor alignment of the hits, a good spatial resolution can help in reducing significantly this background.

\section{Real tracks produced by background muons}

This background may result from muons coming from the "wrong" direction, i.e. from the backward direction (BF muons) or from the "right" direction, ie from the volcano direction (FB muons).

BF muons can be rejected by a time of flight measurement. FB muons are not distinguishable from signal muons.

The origin of background FB muons is not completely understood. One possible source could be the volcano edifice itself. A possible source is multiple scattering (MS) in the volcano, in particular affecting muons that exit the volcano with a low energy (see Figure 4). To quantify this background is hard, as in principle a simulation over the entire volcano edifice is required.

A test measurement has been performed at the Showa-Shinzan lava dome [Nishiyama et al. 2014], using two different muon telescopes consisting of nuclear emulsions films with interposed lead plates. Due to the micrometric spatial resolution of the nuclear emulsion, it is possible to discriminate muons with different energies measuring the muon scattering in the lead sheets. It has been shown that by rejecting muons with energy below $2 \mathrm{GeV}$ the background level was reduced and the signal was compatible with the muon flux expected to survive crossing a few hundred meters of rock in the volcano. With a $200 \mathrm{MeV}$ energy cut, the background level was not compatible with any reasonable value of rock density.

For electronic detectors, the approach followed by Tanaka et al. [2014] to reduce the background level consisted in introducing lead and iron layers into a detector consisting of several planes of plastic scintillators. A drastic background reduction was obtained.

\section{Background rejection in MURAVES}

The good spatial resolution $(\approx 3 \mathrm{~mm})$, the large amount of scintillator bars per layer (64), the usage of at least three $\mathrm{X}-\mathrm{Y}$ layers to reconstruct the track, the short coincidence time window (40 ns) and the time resolution ( $1 \mathrm{~ns})$ adequate for a time of flight measurement are expected to reduce significantly most of the backgrounds discussed in the previous sections.

The insertion of lead layers is expected to reject a notable part of the background from low energy muons, the main source of FB background

They introduce an energy threshold through two effects. The first one is that muons below a minimal energy cannot cross the lead. Table 1 shows the minimum energy as a function of lead thickness, in the range $5-50 \mathrm{~cm}$ [Olive et al. 2014]. The second effect is the multiple scattering suffered by the muons that cross the shield, that is strongly correlated to the muon energy and can be used for its estimate.

However, the use of a large amount of lead can be applied only in detector locations where the lead can be transported and installed. For the measurement campaign at the Mt. Vesuvius this will not be a real problem. In fact, the possible sites selected for the muon telescope can be reached by a truck and geolog-

\begin{tabular}{cc}
\hline Lead thickness $(\mathrm{cm})$ & $\mathrm{E} \min (\mathrm{MeV})$ \\
\hline 15 & 300 \\
20 & 350 \\
25 & 400 \\
30 & 450 \\
35 & 550 \\
40 & 600 \\
45 & 650 \\
50 & 750 \\
\hline
\end{tabular}

Table 1. The minimum energy required to a muon to cross a certain thickness of lead. 
$\frac{-}{8}$

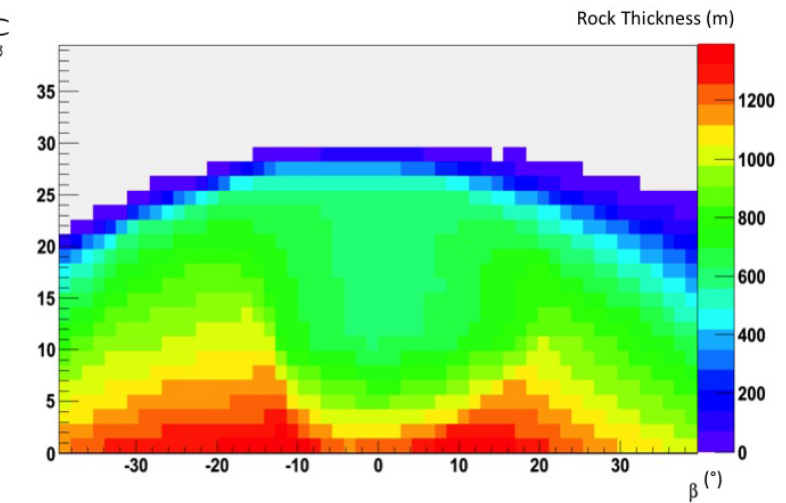

Figure 5. Rock thickness of Mt. Vesuvius as seen from the MURAVES observation point.

ical surveys showed the stability of the ground. In general, one of the main challenges for the next generation of muon telescopes is the introduction of an energy threshold in a more practical way than by using a large amount of lead.

The energy threshold on the muons decreases, of course, the number of signal muons. The expected loss in the signal can be evaluated from the energy spectrum of the signal muon crossing Mt. Vesuvius, as observed from the telescope.

Figure 5 shows the thickness of Mt. Vesuvius, as seen from one of the possible detector sites. As an example, Figure 6 shows the energy spectrum after 1012 $\mathrm{m}$ of rock at an elevation angle of $6^{\circ}$. Figures 7,8 and 9 show the fraction of muons surviving after propagation through volcano that arrive at detector with energies above the energy threshold, in three particular cases corresponding to rock thicknesses of $400 \mathrm{~m}, 800 \mathrm{~m}$ and $1310 \mathrm{~m}$. Figure 10 shows this fraction as a function of elevation and azimuth angles, for two different energy cuts of $1 \mathrm{GeV}$ and $2 \mathrm{GeV}$.

Muons have been generated assuming the energy distribution described in Tanaka et al. [2003].

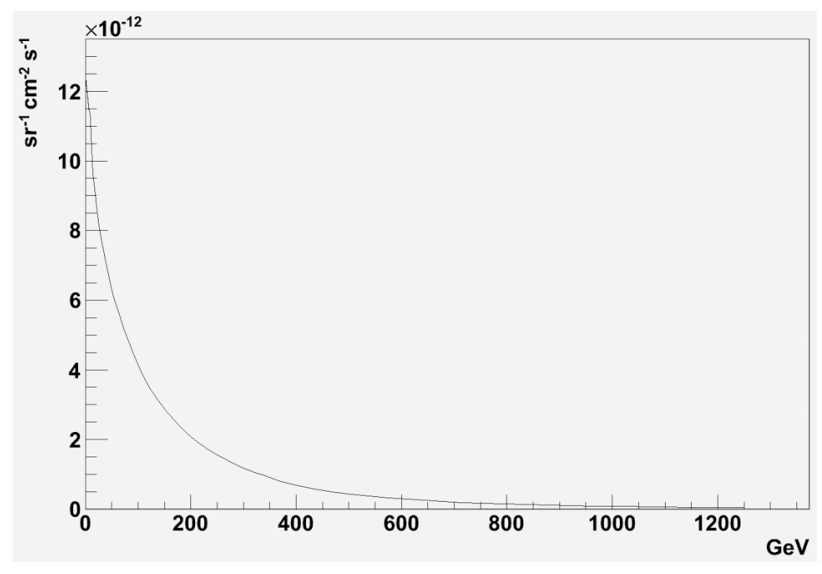

Figure 6. Example of an energy spectrum of muons exiting from the Vesuvio after about $1012 \mathrm{~m}$ of rock thickness and an elevation angle of $6^{\circ}$. Muons have been generated according to the momenta distribution described in Tanaka et al. [2003].

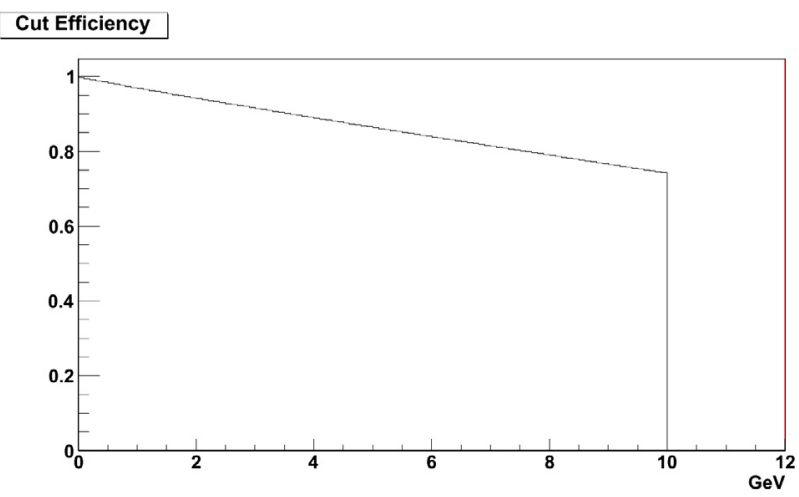

Figure 7. Efficiency of the muon selection as function of the energy cut in the case of $400 \mathrm{~m}$ of rock thickness.

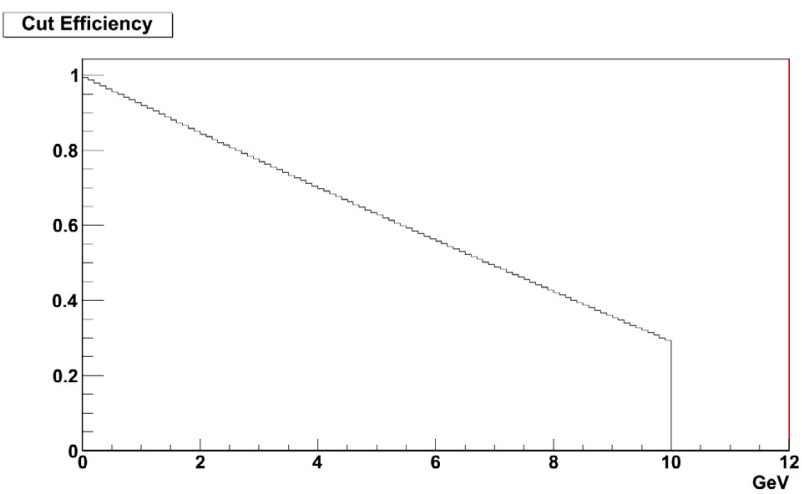

Figure 8. Efficiency of the muon selection as function of the energy cut in the case of $800 \mathrm{~m}$ of rock thickness.

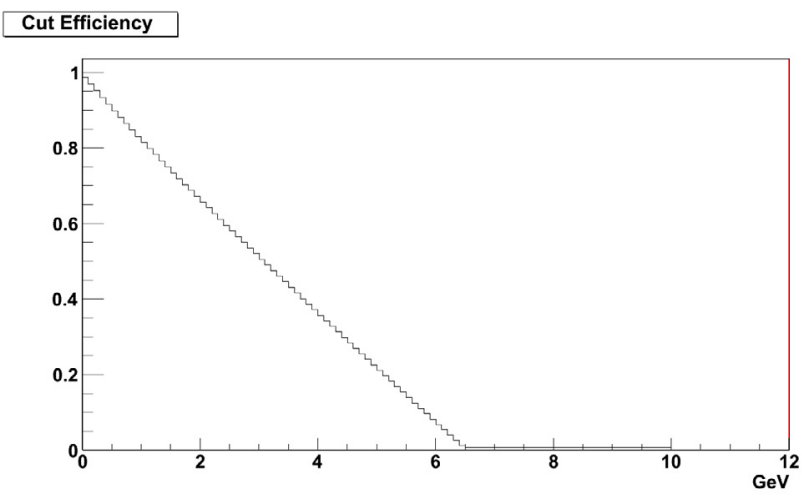

Figure 9. Efficiency of the muon selection as function of the energy cut in the case of $1300 \mathrm{~m}$ of rock thickness.

\section{Rejection of low energy muons in MURAVES}

We have studied a basic detector configuration consisting of three X-Y planes, with a lead layer between the second and the third X-Y plane further downstream with respect to the muon direction (Figure 11). The first two X-Y planes define the muon track, before multiple scattering in the lead. The displacement of in the third X-Y plane with respect to the track projection is correlated to multiple scattering.

In a preliminary study we have used as a multiple scattering estimator the $\chi^{2}$ of the alignment of the three hits. Figure 12 gives the selection efficiency as 

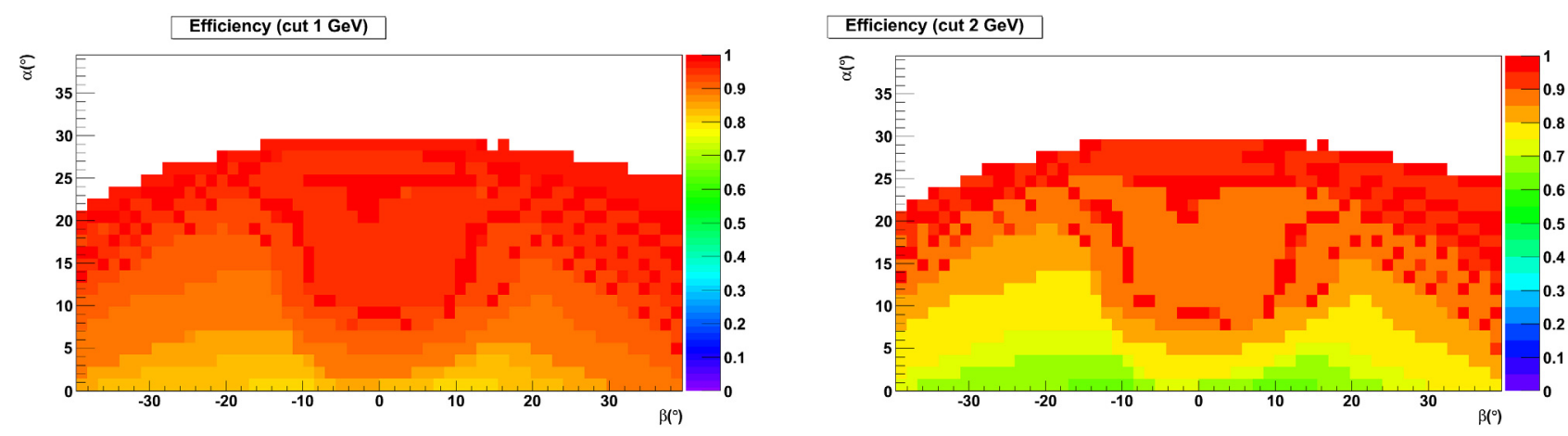

Figure 10. Map of the efficiency of selection of signal muons for a energy cut of $1 \mathrm{GeV}$ (left) and $2 \mathrm{GeV}$ (right).

a function of the muon energy, for lead thicknesses of 25 and $50 \mathrm{~cm}$. In both cases the $\chi^{2}$ cut has been set so that the efficiency for muon with energy of $2 \mathrm{GeV}$ is $95 \%$.

We are considering the possibility of adding a fourth X-Y plane downstream of the third (Figure 13) in order to reconstruct the muon track before and

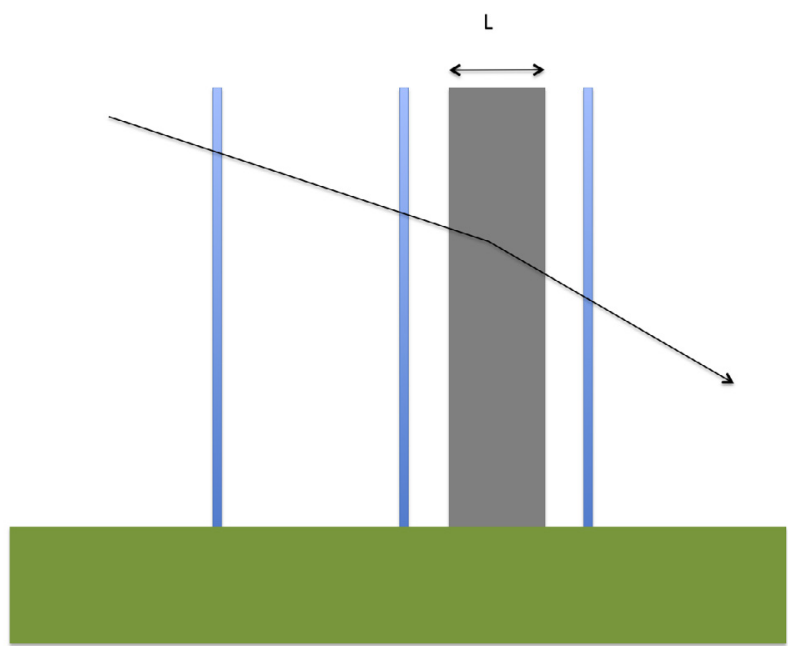

Figure 11. Pictorial view of the placement of the X-Y planes (blue rectangles) and the lead shield (gray rectangle) in the case of three $\mathrm{X}$-Y planes per detector. Signal muons from the volcano are coming from the left (black arrow).

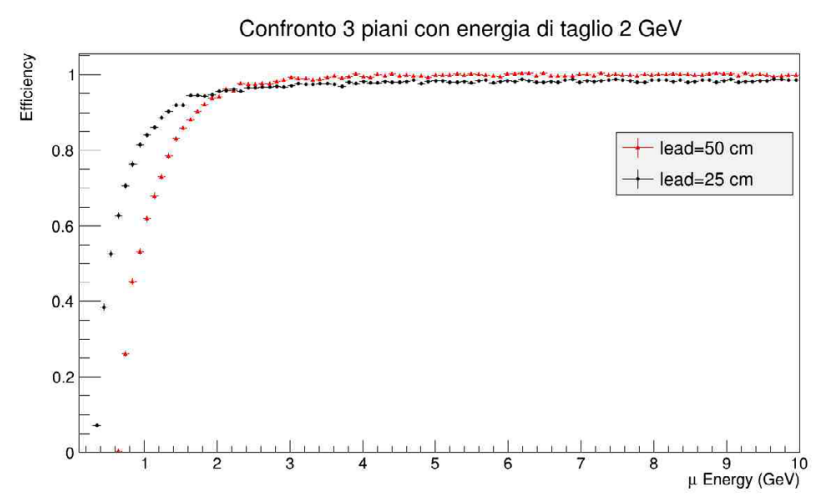

Figure 12. Selection efficiency for muons in the case of two different lead thickness. The cut is based on the chi-square of the track fit and is such that at $2 \mathrm{GeV}$ the efficiency is of $95 \%$. after the lead, so as to measure the distance of closest approach of the two tracks and the scattering angle. Figure 14 gives the muon efficiency versus energy for 25 and $50 \mathrm{~cm}$ lead thickness. The cuts on the two above variables are such that the efficiency is, for a muon of $2 \mathrm{GeV}$, the $95 \%$ for the $25 \mathrm{~cm}$ thickness and $90 \%$ for the $50 \mathrm{~cm}$.

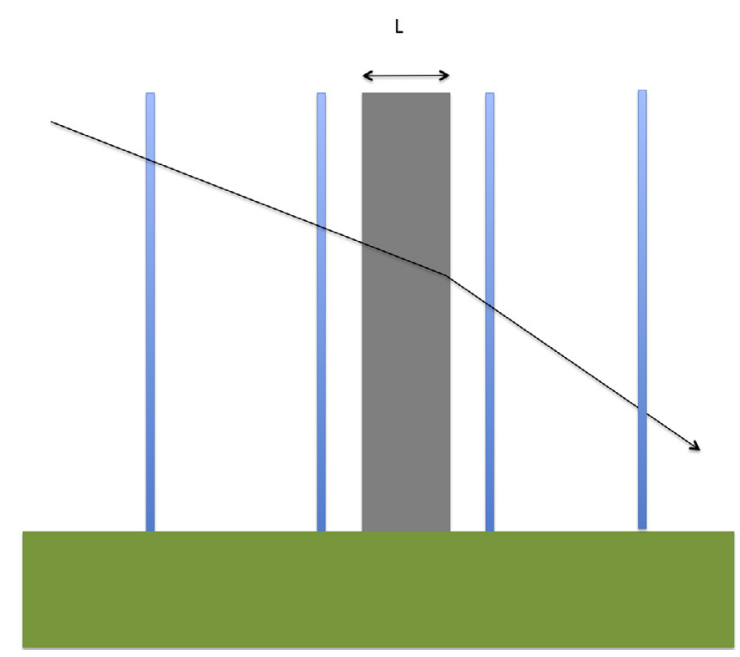

Figure 13. Pictorial view of the placement of the X-Y planes (blue rectangles) and the lead shield (gray rectangle) in the case of four XY planes per detector. Signal muons from the volcano are coming from the left (black arrow).

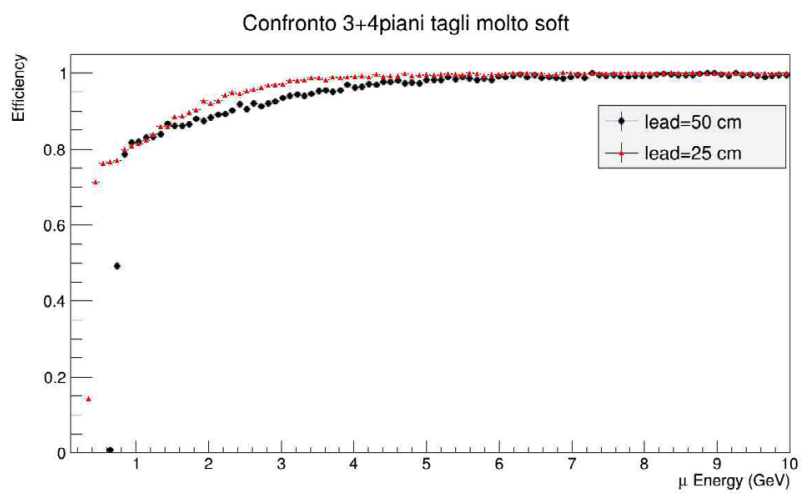

Figure 14. Selection efficiency for muons in the case of two different lead thickness. The cut is based on the chi-square of the track fit and is such that at $2 \mathrm{GeV}$ the efficiency is the $95 \%$. 


\section{Conclusions}

The MURAVES project aims to the first muographic observation of the upper part of the Mt. Vesuvius. An array of four muon detectors of $1 \mathrm{~m}^{2}$ sensitive area is under construction, upgrading the technology developed in the frame of the MU-RAY R\&D program. The use of three or four X-Y tracking planes, the high segmentation and spatial resolution, the measurement of the muon time of flight and the insertion of a lead layer are expected to lead to the required background rejection. However, the complexity of the background is such that the final word will come from experience on the field.

\section{References}

Ambrosi, G., et al. (2011). The MU-RAY project: Volcano radiography with cosmic-ray muons, Nucl. Instrum. Meth. A, 628, 120-123.

Ambrosino, F., et al. (2014). The MU-RAY project: detector technology and first data from Mt. Vesuvius, J. Instrum., 9, C02029.

Ambrosino, F., L. Bonechi, L. Cimmino, R. D’Alessandro, D.G. Ireland, R. Kaiser, D.F. Mahon, N. Mori, P. Noli, G. Saracino, C. Shearer, L. Viliani and G. Yang (2015). Assessing the feasibility of interrogating nuclear waste storage silos using cosmic-ray muons, J. Instrum., 10 (6), T06005.

Anastasio, A., et al. (2013a). The MU-RAY detector for muon radiography of volcanoes, Nucl. Instrum. Meth. A. 732, 423-426.

Anastasio, A., et al. (2013b). The MU-RAY experiment. An application of SiPM technology to the understanding of volcanic phenomena, Nucl. Instrum. Meth. A, 718, 134-137.

Cimmino, L., F. Ambrosino, L. Bonechi, R. Ciaranfi, R. D’Alessandro, V. Masone, N. Mori, P. Noli, G. Saracino, P. Strolin (2017). The MURAVES telescope front-end electronics and data acquisition, Annals of Geophysics, 60 (1), S0104; doi:10.4401/ag-7379.

Nishiyama, R., S. Miyamoto and N. Naganawa (2014). Experimental study of source of background noise in muon radiography using emulsion film detectors, Geosci. Instrum. Meth., 3, 29-39.

Olive, K.A., et al. (2014). Particle Data Group, Chin. Phys. C, 38, 090001.

Tanaka, H., K. Nagamine, N. Kawamura, S.N. Nakamura, K. Ishida and K. Shimomura (2003). Development of a two-fold segmented detection system for near horizontally cosmic-ray muons to probe the internal structure of a volcano, Nucl. Instrum. Meth. A, 507, 657-669.

Tanaka, H.K.M., T. Kusagaya and H. Shinohara (2014). Radiographic visualization of magma dynamics in an erupting volcano, Nat. Commun., 5, 3381.
${ }^{\star}$ Corresponding author: Giulio Saracino, Istituto Nazionale di Fisica Nucleare (INFN), Sezione di Napoli, Naples, Italy; email: saracino@na.infn.it.

C 2017 by the Istituto Nazionale di Geofisica e Vulcanologia. All rights reserved. 